\title{
Coherent Joint-Processing CoMP in Pico-Cellular Lamp-Post Street Deployment
}

\author{
Dragan Samardzija \\ Bell Laboratories, Alcatel-Lucent \\ 791 Holmdel-Keyport Road, \\ Holmdel, NJ 07733, USA \\ Email: dragan.samardzija@alcatel-lucent.com
}

\author{
Andrej Domazetovic \\ University of Novi Pazar \\ Vuka Karadzica bb \\ 36300 Novi Pazar, Serbia \\ Email: adomazetovic@np.ac.rs
}

\begin{abstract}
In this paper we study the performance of coherent joint-processing coordinated multi-point (CoMP) transmission, also known as Network MIMO, in pico-cellular street deployments with lamp-post base station installation. Previously published and experimentally confirmed short-range wireless propagation models have been applied. The performance is compared against the equivalent case of macro-cellular deployment. In both deployments, we report significant Network MIMO gains compared to the conventional solution without coordination. Effects of the base station antenna elevation and transmit power are also analyzed. Greater benefit of Network MIMO is determined in pico- than in macro-cellular systems.
\end{abstract}

\section{INTRODUCTION}

Due to reuse-1, modern communication systems are interference-limited. The interference that originates from the neighboring cells is significantly stronger than the background noise. Considering that the interference is generated by the wireless system itself, a smart coordination of individual transmissions, i.e., coordinated multi-point (CoMP) within the system could result in the interference mitigation, and consequently in the improvement of signal-to-interferenceand-noise ratio (SINR) and achievable data rates. Therefore, the future releases of 3GPP Long Term Evolution (LTE), i.e., LTE-Advanced are to introduce different CoMP techniques [1].

Various multiple antenna techniques, including multipleinput/multiple-output (MIMO), have been shown to significantly improve wireless system spectral efficiency [2]. The original MIMO idea is a link-level solution, for example, considering communication between a single mobile terminal and its dedicated base station. Recent studies also showed that a similar idea could be applied to a network of base stations communicating with multiple terminals. This technique is known as Network MIMO, or CoMP with joint processing and coherent combining [3]-[6]. By applying this technique, what would otherwise present the interference coming from multiple transceivers can become a useful signal and aid reception.

The previously published studies have evaluated the gains achievable by Network MIMO in macro-cellular environments [3], [4]. Sizable gains have been reported in heavily interference-limited systems. In this study we are exploring the benefits of Network MIMO in pico-cellular environments, with lamp-post installations as suggested in [6].

We show that Network MIMO provides higher spectral efficiency gains in the pico-cellular scenarios compared to the corresponding macro-cellular case. Effects of the base station antenna elevation and transmit power are also analyzed.

In Section II we present the system model and Network MIMO processing details. In Section III we describe the simulation methodology and present the corresponding results. We also compare the achievable data rate gains and address the effects of system parameters. We conclude in Section IV.

\section{SYSTEM MODEL}

We consider a street deployment of base stations with antennas mounted on lamp posts. We focus on 1) urban environment streets assuming large buildings - reflectors present on both sides of the road with trees and bushes, as seen in towns and cities, 2) rural environment streets assuming no large objects or structures in the vicinity of the road, as seen in open roadways, country roads or parks, and 3) a typical parking lot with lanes of parked cars as seen in front of shopping malls or office buildings. The base station antennas are connected via a wired backhaul network to the Network MIMO controller where the uplink processing and downlink preprocessing is performed [7]. A frequency reuse1 network is analyzed where all base stations and terminals communicate over a single frequency. In this analysis we assume, without loss of generality, that each terminal is associated with a unique base station, i.e., one terminal per each base station coverage area. To avoid the edge effects a wrapped-around linear topology is used where movement of mobile terminals and electro-magnetic radiation is bound to a circle. Correspondingly, in the macro-cellular case this linear topology may model base station deployment along a highway, with vehicular mobile terminals.

To analyze Network MIMO performance in pico-cellular deployments we use short-range wireless channel models that are originally introduced in [8]. Three channel models are derived and validated by measurements, each corresponding to urban environment, rural environment, and a typical parking lot. The models are results of a hybrid approach combining 
deterministic and stochastic techniques. The deterministic raytracing technique is used to model the main modes of propagation. In addition, the stochastic technique is used to model the remaining, lower power scatter components. The models include effects like antenna patterns and variations in reflection coefficients with respect to incident wave angles and polarity. Considering the above features, the models are particularly suitable for the street deployments which are the focus of this study.

Note that the applicability of the above models is limited to the cases with the direct line-of-sight (LOS) between the base station and a mobile terminal, thus excluding shadowing effects. In addition, the stochastic component is experimentally validated up to 20 meters from the base station and analytically extrapolated for larger distances.

Since we will compare the Network MIMO performance in pico-cellular versus macro-cellular deployments, we use a well-established macro-cellular path loss model in [9] (the modified COST 231 Hata model, page 40, equation 6). In addition to the path loss, log-normal shadowing with $8 \mathrm{~dB}$ standard deviation and Rayleigh fading have been applied.

\section{A. Uplink}

We consider a Network MIMO coordination cluster with $M$ base stations and $N$ mobile terminals, for $M \geq N$. Without loss of generality, we assume a single-antenna base stations and terminals. The uplink signal received by all base stations is $\mathbf{y}_{\mathbf{u}}=\mathbf{H}_{\mathbf{u}} \mathbf{x}_{\mathbf{u}}+\mathbf{n}_{\mathbf{u}}$, where $\mathbf{y}_{\mathbf{u}}$ is an $M$-dimensional vector; $\mathbf{H}_{\mathbf{u}}$ is the MIMO channel matrix (entry $h_{u(m, n)}$ is the complex gain between mobile terminal $n$ and base station $m$, for $n=$ $1, \cdots, N$ and $m=1, \cdots, M) ; \mathbf{x}_{\mathbf{u}}$ is an $N$-dimensional vector, whose $n$-th component corresponds to a signal transmitted by terminal $n$ and $\mathbf{n}_{\mathbf{u}}$ is a complex, zero-mean additive white Gaussian noise vector, of variance $N_{o}, E\left[\mathbf{n}_{\mathbf{u}} \mathbf{n}_{\mathbf{u}}{ }^{\mathbf{H}}\right]=N_{o} \mathbf{I}$. In the Network MIMO controller, the received signal is spatially filtered using the matrix $\mathbf{A}$

$$
\mathbf{A}=\left(\mathbf{H}_{\mathbf{u}}^{\mathbf{H}} \mathbf{H}_{\mathbf{u}}\right)^{-\mathbf{1}} \mathbf{H}_{\mathbf{u}}^{\mathbf{H}},
$$

which is a standard zero-forcing matrix [3]. The above spatial filter $\mathbf{A}$ will diagonalize the channel $\mathbf{H}_{\mathbf{u}}$, and consequently spatially orthogonalize the uplink transmissions that originate from different mobile terminals within the coordination cluster. In other words, the cluster uplink intercell interference will be removed (projected away) at the expense of noise amplification. After the filtering, the uplink SINR for terminal $i$ is

$$
\operatorname{SINR}_{\mathbf{u} i}=\frac{P_{\mathbf{u} i}}{N_{o} \sum_{j=1, \cdots, M}\left|a_{(i, j)}\right|^{2}}
$$

where $P_{\mathbf{u}_{i}}$ is the transmitted uplink signal power of the $i$-th terminal, and $a_{(i, j)}$ is the $i$-th row $j$-th column entry of the matrix A.

In order for the Network MIMO controller to perform the above filtering, it must have a knowledge of the channel state. In this analysis we assume perfect knowledge of the uplink channel state.
Note that instead of the above zero-forcing spatial filter, a minimum mean square error (MMSE) filter may be applied. However, it requires an estimate of the interference and noise covariance matrix, which is a subject of our ongoing work.

\section{B. Downlink}

The downlink MIMO channel matrix is $\mathbf{H}_{\mathbf{d}}=\mathbf{H}_{\mathbf{u}}^{\mathbf{T}}$. Unlike the base stations, the mobile terminals are not inter-connected via a wired backhaul network. The transmitted downlink signals that are sent to individual terminals are preprocessed by the Network MIMO controller. Namely, a spatial pre-filter $\mathbf{W}$ is applied as

$$
\mathbf{z}_{\mathbf{d}}=\mu \mathbf{W} \mathbf{x}_{\mathbf{d}}
$$

where $\mathbf{x}_{\mathbf{d}}$ is an $N$-dimensional vector, whose $n$-th component is a data-carrying signal dedicated to mobile terminal $n$. In addition, $\mathbf{z}_{\mathbf{d}}$ is an $M$-dimensional transmit vector, whose $m$ th component is a signal transmitted from the $m$-th base station antenna in the cluster. In this study we apply the pre-filter $\mathbf{W}$

$$
\mathbf{W}=\mathbf{H}_{\mathbf{d}}^{\mathbf{H}}\left(\mathbf{H}_{\mathbf{d}} \mathbf{H}_{\mathbf{d}}^{\mathbf{H}}\right)^{-\mathbf{1}} \mathbf{D},
$$

where $\mathbf{D}$ is a $N \times N$ diagonal matrix. Before we define $\mathbf{D}$, we will address the downlink power optimization, which is not present in the uplink case.

Certain mobile terminals will experience either low or high channel quality (e.g., depending how far they are from the base stations). If the matrix $\mathbf{D}$ had identical diagonal entries, the spatial pre-filter would truly equalize the effective channel gains among different mobile terminals. Consequently, in order to improve data rates for terminals with lower channel quality, the data rates of terminals with higher channel quality will be reduced and overall system throughput will decrease. Instead of identical diagonal entries of $\mathbf{D}$, in the following we consider a solution that successfully solves the above problem.

The problems of power optimization and system throughput maximization have been studied in [10]. Computationally intensive solutions have been proposed. We consider an effective and simplified power optimization solution that was implemented and evaluated in [6]. We apply the weighting factor to each terminal which is proportional to its channel quality (measured as sum of path gains from all base station antennas to mobile terminal antenna). Consequently, even after the spatial pre-filtering, terminals with higher channel quality will maintain proportionally higher data rates. To achieve the above, the $i$-th diagonal entry of $\mathbf{D}$ is defined as

$$
d_{(i, i)}=\sqrt{\sum_{j=1, \cdots, M}\left|h_{d(i, j)}\right|^{2}},
$$

$h_{d(i, j)}$ is the $i$-th row $j$-th column entry of the channel matrix $\mathbf{H}_{\mathbf{d}}$.

In this study we assume that there is a maximum transmit power $P_{\max }$ that each antenna is allowed to radiate. This is known as per-antenna power constraint. The scaling factor in (3), $\mu$, that guarantees the above constraint is given as

$$
\mu=\sqrt{\frac{P_{\max }}{\xi}},
$$




$$
\xi=\max _{i=1, \cdots, M}\left(\sum_{j=1, \cdots, N}\left|w_{(i, j)}\right|^{2}\right),
$$

where $w_{(i, j)}$ are elements of pre-filter matrix $\mathbf{W}$. Based on the above, the downlink SINR for terminal $i$ is

$$
\operatorname{SINR}_{\mathrm{d} i}=\frac{\mu^{2} d_{(i, i)}^{2}}{N_{o}}
$$

In order for the Network MIMO controller to perform the above pre-filtering, it must have a knowledge of the downlink channel state. As in the uplink case, we assume perfect knowledge of the downlink channel state.

\section{Simulation methodology AND RESUlts}

The objective of our simulations is to determine cumulative distribution functions (CDFs) of the achievable uplink and downlink data rates, i.e., spectral efficiency. Each base station has a single terminal positioned within its coverage area. The distance between the base station antenna and the corresponding terminal is drawn from a uniform distribution subject to a constraint that the terminal cannot be placed closer to the antenna than a predefined minimum distance $d_{\text {min }}$. Once all the terminals are placed, the channel realizations between each terminal and base station antennas are determined (i.e., entries of $\mathbf{H}_{\mathbf{u}}$ and $\mathbf{H}_{\mathbf{d}}$ ). From these channel realizations, we determine SINR for each mobile terminal. We considered four different situations: a) no interference (i.e., system consisting of a single mobile terminal and base station, as a baseline for comparisons), b) no Network MIMO coordination, c) Network MIMO for the uplink, and d) Network MIMO for the downlink. From the obtained SINR, the spectral efficiency is calculated using Shannon's capacity formula.

We have studied several parameters that influence system performance. In pico- and macro-cellular cases, the parameters are selected to ensure a fair Network MIMO performance comparison. The transmit power is selected such that the edge SNR is $S N R_{\text {edge }}=20 \mathrm{~dB}$ (ratio between received signal power and $N_{o}$ in absence of interference and small scale fading effects). Accordingly, the cell size becomes directly related to the transmit power and is no longer a free parameter. In the pico-cellular case, the minimum distance is set to $d_{\min }=1.5$ $\mathrm{m}$ to be $10 \%$ of the cell radius. In the macro-cellular case $d_{\min }=50 \mathrm{~m}$, which is the path loss model limitation [9] and also $10 \%$ of the cell radius. The distances between neighboring base station antennas are consequently $30 \mathrm{~m}$ and $1000 \mathrm{~m}$ in the pico- and macro-cellular cases, respectively. In the macrocellular case we also evaluated performance for $d_{\min }=250$ $\mathrm{m}$ and intercell distance of $5000 \mathrm{~m}$, but as expected, the results were identical. The carrier frequency is $f_{c}=5.3$ $\mathrm{GHz}$ being unlicensed and attractive for pico-cellular systems. In addition, that particular frequency was also used in the channel models and corresponding propagation measurements in [8]. The channel bandwidth is $20 \mathrm{MHz}$ for the noise power calculations. Simulations were also performed for lower carrier frequencies. What became evident is that higher frequencies impose a constant power penalty in the system. Therefore, the effects of the cell size and carrier frequency have just been reduced to the scaling factor of the transmit power (due to the fixed cell-edge SNR).

Once the above parameters are set, we first investigate the choice for base station antenna elevation in the picocellular case. The mobile terminal antenna is in all cases at $1.5 \mathrm{~m}$. Since the models in [8] include the antenna radiation patterns, from simulations (confirmed by measurements in [8]) we conclude that by increasing the base station antenna elevation, a terminal may often enter the radiation null-zone. Additionally, an exponential decay in received power with the distance is most notable in the proximity of the base station antenna since the relative path differences are higher when paths are short. Due to these two effects, path loss is higher with increased antenna elevation. At larger distances, the effect of base station antenna elevation is negligible. This is depicted in Figure 1, where we present the path loss as a function of distance. Two sets of curves are presented - one not including antenna patterns, and the other including the omnidirectional antenna patterns for both the base station and the mobile terminal. In the figure, the former case is offset by $-30 \mathrm{~dB}$ for easier comparison. These effects, evident in the first $10 \mathrm{~m}$ around the base station antenna, lead to the choice for base station antenna elevation of $1.8 \mathrm{~m}$, or generally, as close to the terminal antenna elevation as practically possible. The presented results are for the street in rural area. However, for other models the results are almost identical.

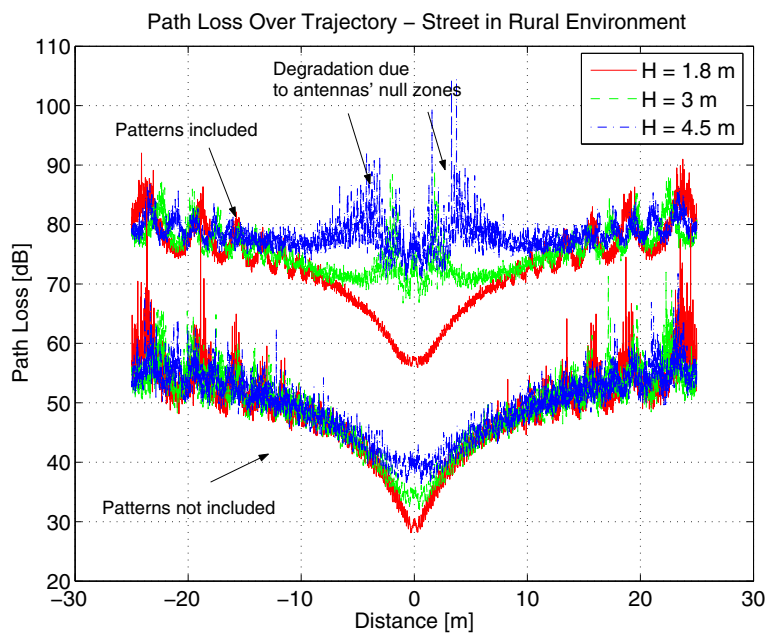

Fig. 1. Path loss as a function of the base station antenna elevation. The case where antenna patterns are not included is offset by $-30 \mathrm{~dB}$.

Having set the base station antenna elevation to $1.8 \mathrm{~m}$, we choose $7 \times 7$ system $(M=7$ single-antenna base stations, and $N=7$ single-antenna mobile terminals) and determine the spectral efficiency for all pico-cellular models. The results are presented in Figure 2. We note that in all three types of pico-cellular environments, the Network MIMO gains are approximately the same. The uplink shows higher gains since it is not limited by the per-antenna power constraint as the downlink is. The benefit of Network MIMO is obvious - for 
the 5-percentile cases, the spectral efficiency is improved from $1 \mathrm{~b} / \mathrm{s} / \mathrm{Hz}$ to $4 \mathrm{~b} / \mathrm{s} / \mathrm{Hz}$ for the downlink, and $6 \mathrm{~b} / \mathrm{s} / \mathrm{Hz}$ for the uplink. The median value is increased from $2 \mathrm{~b} / \mathrm{s} / \mathrm{Hz}$ to 8 and $9 \mathrm{~b} / \mathrm{s} / \mathrm{Hz}$, respectively.

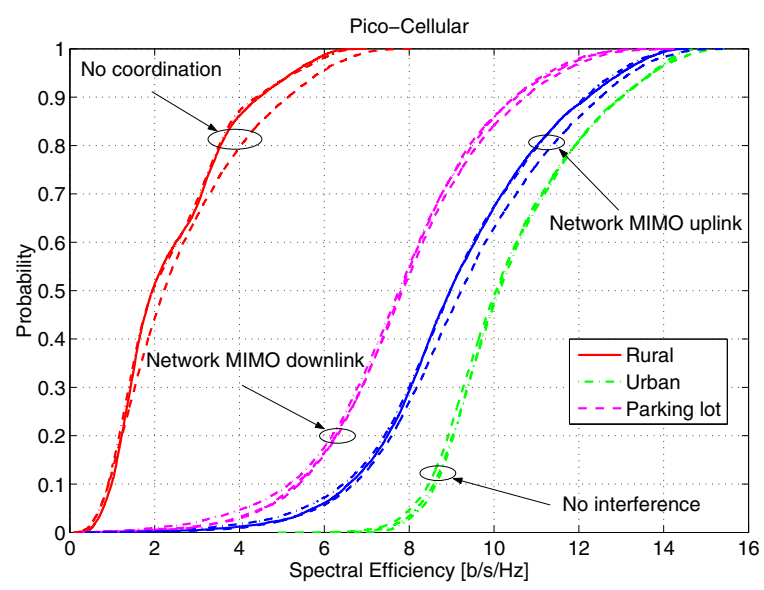

Fig. 2. Spectral efficiency for pico-cellular deployment $(7 \times 7$ system $)$.

Furthermore, the performance is compared against the macro-cellular deployment. The parameters are chosen as in the pico-cellular case. Namely, the same carrier frequency, channel bandwidth, and edge SNR have been used in both cases. The cell radius is chosen to be 10 times larger than the model's cutoff distance $d_{\min }=50$, thus the cell size is 500 $\mathrm{m}$ (this is to match $d_{\min }=1.5 \mathrm{~m}$ and $15 \mathrm{~m}$ cell size in the pico-cellular case). The resulting CDF is shown in Figure 3. It clearly shows that the gains are smaller than in the picocellular deployments, especially for the downlink. This is a consequence of 3.5 macro-cellular path loss exponent versus the pico-cellular free-space square law path loss. To verify this claim, we have set the exponent in the macro-cellular model to 2 obtaining results identical to the pico-cellular case.

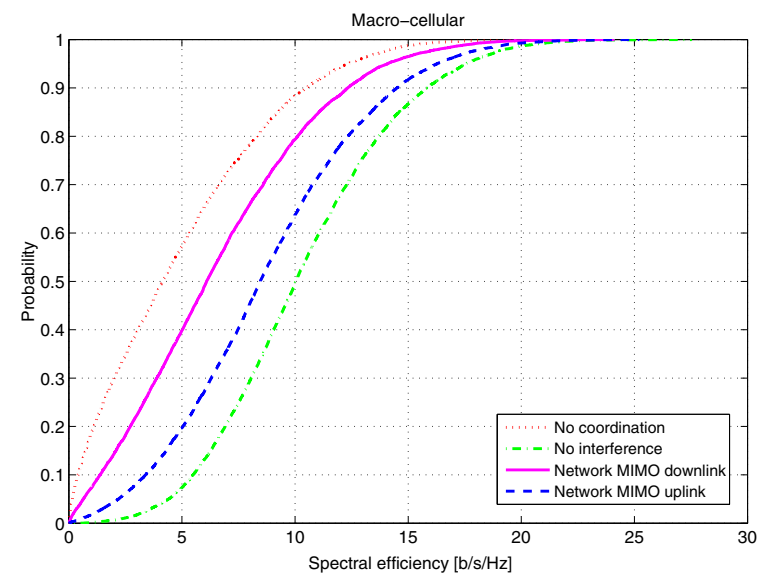

Fig. 3. Spectral efficiency for macro-cellular roadway deployment $(7 \times 7$ system), linear topology.

The spectral efficiency gains for all cases are summarized in Figures 4 and 5. The relative gains of Network MIMO are more pronounced in pico- than in macro-cellular deployments.

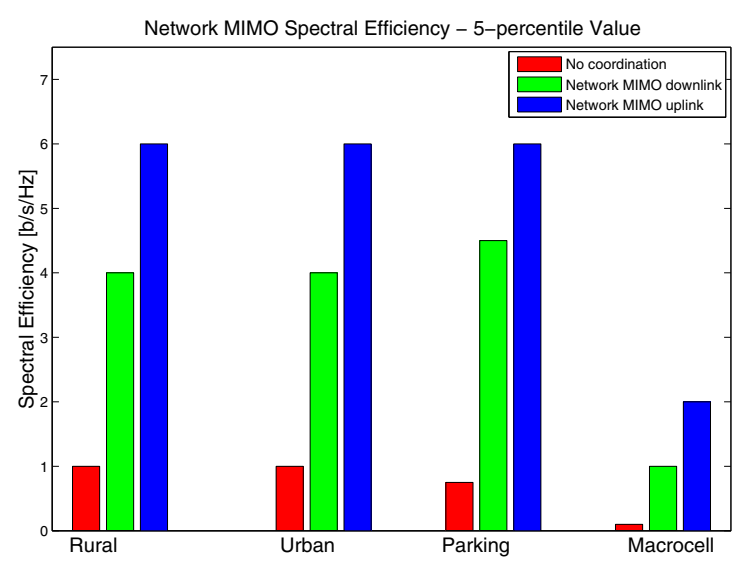

Fig. 4. Spectral efficiency, 5 -percentile case $(7 \times 7$ system $)$, linear topology.

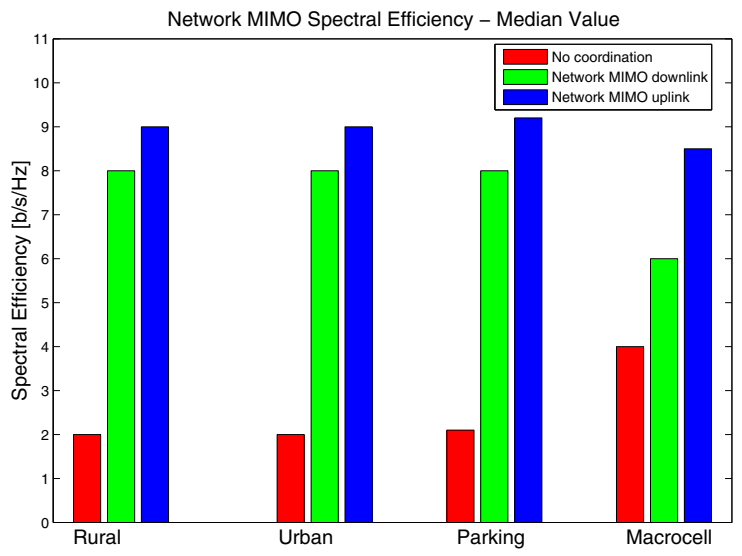

Fig. 5. Spectral efficiency, median value $(7 \times 7$ system $)$, linear topology.

Finally, note that the transmit power levels used in picocellular simulations are very low - order of milliwatts. Therefore, further increase in system capacity could be accomplished by higher transmit power. For example, considering a conventional power amplifier technologies hundreds of milliwatts of transmit power can be efficiently achieved. In Figure 6 we present SINR for three different transmit power levels (-10 $\mathrm{dBm}, 5 \mathrm{dBm}$ and $20 \mathrm{dBm}$ ), indicating significant gains with the power increase. However, the base station coordination, like Network MIMO, is needed to enable the improvements due to higher transmit power (e.g, in Figure 6 the case without coordination is not affected by the increase in transmit power level as the interference is also amplified). Other three curves are shifted by $15 \mathrm{~dB}$ following the amount of power increase. In the macro-cellular case, the transmit power levels are much higher, therefore, limiting how much further they could be 
increased. This is another aspect pointing to a greater benefit of Network MIMO in pico- than in macro-cellular systems.

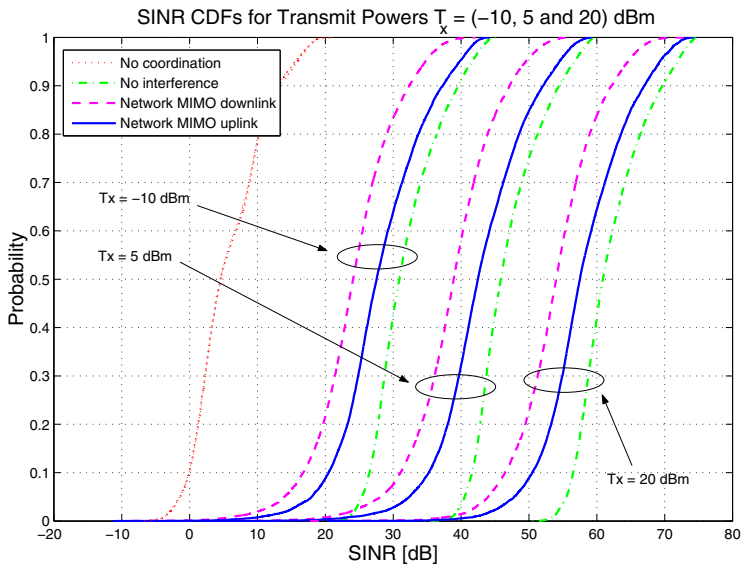

Fig. 6. SINR with three different transmit power levels $T_{x}=(-10,5,20)$ $\mathrm{dBm}(7 \times 7$ system $)$.

It is worth noting that the current transmitter and receiver commercial implementations limit the maximum achievable SINR to approximately $30 \mathrm{~dB}$. Therefore, the values in Figure 6 that significantly exceed $30 \mathrm{~dB}$ are currently impractical due to the commercial technology limitations. Nevertheless, we present those results to illustrate potentials of Network MIMO.

\section{CONCLUSION}

In this study we have analyzed the coherent joint-processing CoMP, i.e., Network MIMO applicability and performance in pico-cellular environments - namely streets with base station antennas mounted on lamp posts. We have shown that the elevation of the base station antennas should be as close to the elevation of the mobile terminals as practically possible. We have demonstrated significant Network MIMO gains in terms of downlink and uplink spectral efficiency over conventional case without any coordination. Providing that the system is in a interference-limited regime, we have shown that the carrier frequency and cell size do not affect the performance (since the transmit power is scaled to guarantee the cell edge SINR). Significantly higher Network MIMO gains have been observed in the pico-cellular than in the macro-cellular deployments. In addition, when Network MIMO is employed, we have shown that with a small increase in absolute transmit power, picocellular system capacity can be further increased.

Although not a focus of this study, Network MIMO may be more easily implemented in pico- than in macro-cellular systems. For example, smaller distances within the coordination cluster do not impose a need for longer cyclic prefix in OFDM (e.g., as in LTE). Shorter backhaul transport links between the base stations result in lower latencies which is essential for better synchronization and ability to track channel changes. Furthermore, pico-cellular deployments are expected to serve pedestrian users with lower mobility, thus providing higher quality of the uplink and downlink channel state information.

The above results and discussions strongly recommend application of Network MIMO in pico-cellular systems.

\section{REFERENCES}

[1] "Feasibility Study for Further Advancements for E-UTRA (LTEAdvanced)," 3GPP TR 36.912, 2009.

[2] G. J. Foschini and M. J. Gans, "On limits of wireless communications in a fading environment when using multiple antennas," Wireless Personal Communications, no. 6, pp. 315-335, 1998.

[3] M. K. Karakayali, G. J. Foschini, and R. A. Valenzuela, "Network coordination for spectrally efficient communications in cellular systems," IEEE Transactions on Wireless Communications, vol. 13, pp. 56-61, August 2006.

[4] S. Venkatesan, "Coordinating base stations for greater uplink spectral efficiency in a cellular network," International Symposium on Personal, Indoor, Mobile Radio Communications, pp. 1-5, September 2007.

[5] S. Venkatesan, A. Lozano, R, and Valenzuela, "Network MIMO: overcoming intercell interference in indoor wireless systems," The Annual Conference on Signals, Systems and Computers, pp. 83-87, November 2007.

[6] D. Samardzija, H. Huang, R. Valenzuela, and T. Sizer, "An experimental downlink multiuser MIMO system with distributed and coherentlycoordinated transmit antennas," IEEE International Conference on Communications, 2007.

[7] H. Huang and D. Samardzija, "Determining backhaul bandwidth requirements for Network MIMO," The European Signal Processing Conference EUSIPCO, August 2009.

[8] A. Domazetovic, L. Greenstein, I. Seskar, and N. Mandayam, "Propagation models for short-range wireless channels with predictable path geometries," IEEE Transactions on Communications, vol. 53, pp. 1123 1126, July 2005.

[9] R. Srinivasan and et. at., "IEEE $802.16 \mathrm{~m}$ Evaluation Methodology Document (EMD)," IEEE 802.16 Broadband Wireless Access Working Group, 2009.

[10] F. Boccardi and H. Huang, "Zero-forcing precoding for the MIMO broadcast channel under per-antenna power constraints," IEEE Workshop on Signal Processing Advances in Wireless Communications, July 2006. 